\title{
Blood flow measurements in rats using four color microspheres during blockade of different vasopressor systems
}

\section{K. De Angelis ${ }^{1,4}$, \\ V.M. Gama ${ }^{1,2}$, \\ V.A.M. Farah ${ }^{3}$ and \\ M.C. Irigoyen ${ }^{1,2}$}

\section{Correspondence \\ M.C. Irigoyen \\ Unidade de Hipertensão \\ Instituto do Coração, FM, USP \\ Av. Enéas C. Aguiar, 44 \\ 05403-000 São Paulo, SP \\ Brasil \\ Fax: +55-11-3069-5048 \\ E-mail: hipirigoyen@incor.usp.br}

Research supported by FAPESP,

Fundação Zerbini, and CNPq.

......................

Received March 29, 2004

Accepted October 7, 2004

\author{
${ }^{1}$ Unidade de Hipertensão, Instituto do Coração, Universidade de São Paulo, \\ Faculdade de Medicina, São Paulo, SP, Brasil \\ ${ }^{2}$ Departamento de Nefrologia, Escola Paulista de Medicina, \\ Universidade Federal de São Paulo, São Paulo, SP, Brasil \\ 3 UNIPESQ, Universidade de Santo Amaro, Santo Amaro, SP, Brasil \\ ${ }^{4}$ Laboratório do Movimento Humano, Universidade São Judas Tadeu, \\ São Paulo, SP, Brasil
}

\section{Abstract}

The use of colored microspheres to adequately evaluate blood flow changes under different circumstances in the same rat has been validated with a maximum of three different colors due to methodological limitations. The aim of the present study was to validate the use of four different colors measuring four repeated blood flow changes in the same rat to assess the role of vasopressor systems in controlling arterial pressure (AP). Red $(150,000)$, white $(200,000)$, yellow $(150,000)$, and blue $(200,000)$ colored microspheres were infused into the left ventricle of 6 male Wistar rats 1) at rest and 2) after vasopressin (aAVP, $10 \mu \mathrm{g} / \mathrm{kg}, i v), 3$ ) renin-angiotensin (losartan, 10 $\mathrm{mg} / \mathrm{kg}, i v$ ), and 4) sympathetic system blockade (hexamethonium, 20 $\mathrm{mg} / \mathrm{kg}, i v)$ to determine blood flow changes. AP was recorded and processed with a data acquisition system (1-kHz sampling frequency). Blood flow changes were quantified by spectrophotometry absorption peaks for colored microsphere components in the tissues evaluated. Administration of aAVP and losartan slightly reduced the AP (-5.7 \pm 0.5 and $-7.8 \pm 1.2 \mathrm{mmHg}$, respectively), while hexamethonium induced a $52 \pm 3 \mathrm{mmHg}$ fall in AP. The aAVP injection increased blood flow in lungs (78\%), liver (117\%) and skeletal muscle (>150\%), while losartan administration enhanced blood flow in heart (126\%), lungs $(100 \%)$, kidneys $(80 \%)$, and gastrocnemius (75\%) and soleus (94\%) muscles. Hexamethonium administration reduced only kidney blood flow $(50 \%)$. In conclusion, four types of colored microspheres can be used to perform four repeated blood flow measurements in the same rat detecting small alterations such as changes in tissues with low blood flow.

Microsphere techniques provide more detailed information about regional perfusion between and within organs than the use of flow probes. Microspheres are also easier
Key words

- Colored microspheres

- Arterial pressure

- Sympathetic system

- Renin-angiotensin

- Vasopressin

- Local blood flow measurement to use and give higher resolution than methods based on molecular tracer washout (1). The use of colored microspheres (CM) represents a simple and safe method, especially 
because it avoids the radiation associated with radioactive spheres (2-4). Frequently, repeated blood flow measurements must be performed in the same animal (same tissue or organ) (5-7). For this purpose, microspheres labeled with different color components are injected, and the components are then measured simultaneously. However, the determination of the amount of the different components may be disturbed by interference between the different absorption spectra. Indeed, CM have been further validated $(4,6,8)$ and employed extensively, although usually with a maximum of three different colors (7). The present study was designed to test the possibility to use four different microsphere colors: white $(370 \mathrm{~nm})$, yellow $(448 \mathrm{~nm})$, red $(530 \mathrm{~nm})$, and blue $(672 \mathrm{~nm})$.

To assess the individual contribution and the interaction of three vasopressor systems in blood pressure control and blood flow distribution we used four repeated estimates in the same rat, i.e., a basal one and three more after vasopressin, renin-angiotensin and sympathetic system blocker injection. Thus, we obtained four repeated blood flow estimates using a combination of different numbers and colors by adapting the standardized infusion protocols, dye extraction procedures and microsphere measurements.

Six male Wistar rats from the Animal House of Universidade de São Paulo, São Paulo, SP, Brazil, weighing $290 \pm 5$ g, received standard laboratory chow ad libitum and were housed in individual cages in a temperature-controlled room $\left(22^{\circ} \mathrm{C}\right)$ with a 12-h dark/light cycle. All surgical procedures and protocols used were in accordance with the Guidelines for Ethical Care of Experimental Animals and Use Committee.

The rats were anesthetized with a mixture of ketamine $(80 \mathrm{mg} / \mathrm{kg})$ and xylazine $(12$ $\mathrm{mg} / \mathrm{kg}$ ). Catheters filled with saline were implanted into the femoral artery and vein and into the left ventricle. PE-10 catheters were positioned into the abdominal aorta and the vena cava by way of the femoral artery and vein for direct measurements of arterial pressure (AP) and for drug injections, respectively. A third PE-50 catheter was inserted into the left ventricle via the right carotid artery for infusion of the CM. The position of the catheter was determined by observing the characteristic left ventricular pressure waveform at surgery and confirmed at autopsy. The catheters were anchored with silk sutures and exteriorized on the back of the neck. Rats receiving food and water ad libitum were studied one day after catheter placement; the animals were conscious and allowed to move freely during the experiments.

The femoral artery cannula was connected to a strain-gauge transducer (Narco Bio-Systems Miniature Pressure Transducer RP 1500, Houston, TX, USA) and blood pressure signals were monitored continuously, except during microsphere infusion and withdrawal of a reference blood sample. The AP signals were recorded and processed with a microcomputer equipped with an analog-to-digital converter board (CODAS, 1-kHz sampling frequency; Dataq Instruments, Inc., Akron, $\mathrm{OH}, \mathrm{USA}$ ). The recorded data were analyzed on a beat-to-beat basis to quantify changes in mean AP and heart rate (HR) as described elsewhere (9).

After 20 min of basal AP recording, a vasopressin V1 receptor antagonist (aAVP, $10 \mu \mathrm{g} / \mathrm{kg}$; Sigma, St. Louis, MO, USA) was administered $i v$ and the AP signals were recorded over a period of $10 \mathrm{~min}$. When AP returned to basal values ( $\cong 25 \mathrm{~min}$ ), losartan, an angiotensin II AT1 receptor antagonist (10 mg/kg; Du Pont Merck, Wilmington, DE, USA), was injected $i v$ and AP was recorded for $10 \mathrm{~min}$. Finally, after a period of recovery ( $\cong 25 \mathrm{~min})$ AP returned to baseline values and hexamethonium, a sympathetic ganglion blocking drug (20 mg/kg; Sigma), was administered $i v$ and blood pressure was recorded over a period of $10 \mathrm{~min}$ (10). The efficacy of blockade was determined by using vasopressin and angiotensin II after aAVP 
and losartan administration, respectively. The responses to vasopressor system blockade were analyzed by averaging mean arterial pressure (MAP) values obtained during a 5to 10-min recording period after the administration of each drug.

Dye-Trak CM (15 $\mu \mathrm{m}$; Triton Technology, San Diego, CA, USA) were infused at rest (red: 150,000 $\mathrm{CM}$ ) and $10 \mathrm{~min}$ after aAVP (white: 200,000 CM), losartan (yellow: $150,000 \mathrm{CM}$ ) and hexamethonium (blue: 200,000 CM) administration for blood flow and cardiac output (CO) determination. Microsphere infusion and processing were performed by the method of Hakkinen et al. (4).

Vials of commercial stocks of microsphere infusates were sonicated for $5 \mathrm{~min}$ and inverted several times immediately before dilution to the desired concentration with $0.9 \%$ saline containing $0.01 \%$ Tween 80 . To determine the average dye content of the microspheres, $200 \mu 1$ of the diluted commercial suspensions was taken after $3 \mathrm{~min}$ of suspension sonication. These aliquots were placed in a $15-\mathrm{ml}$ tube and were centrifuged, dried, and extracted with dimethylformamide (DMF; Sigma). The mean absorbance was determined with a spectrophotometer and divided by the average microsphere concentration to obtain an average dye concentration [absorbance units (spheres $\left.\mathrm{ml}^{-1}\right)^{-1}$ ].

The sphere dilutions were sonicated for 3 min before infusion. A coil of PE-50 tubing $(75 \mathrm{~cm})$ was then filled with $180 \mu 1$ of the infusate of CM and interposed between the left ventricular catheter and a 1-ml syringe containing $0.5 \mathrm{ml}$ of prewarmed $\left(40^{\circ} \mathrm{C}\right) \mathrm{sa}-$ line. This syringe was mounted on a variable speed infusion pump (Infusion Pump 22; Harvard Apparatus, South Natick, MA, USA). With a withdrawal pump (Infusion and Withdrawal Pump; Harvard Apparatus), reference blood samples were drawn from the abdominal aorta catheter at the rate of 0.5 $\mathrm{ml} / \mathrm{min}$ into a pre-heparinized and weighed 1-ml disposable syringe. The withdrawal of a reference blood sample was started $10 \mathrm{~s}$ before the beginning of microsphere infusion and was continued for $75 \mathrm{~s}$. The microspheres and saline were infused for $50 \mathrm{~s}$ at a rate of $0.36 \mathrm{ml} / \mathrm{min}$. After microsphere infusion the animals were killed with a thiopental overdose and heart, lung, kidneys, liver, and the entire gastrocnemius, soleus and brachial triceps muscles were removed to determine regional blood flow.

The reference blood samples and tissues were processed as described by Hakkinen et al. (4). To extract the dyes from the isolated dried microspheres, $250 \mu \mathrm{l}$ DMF was added to each tube, which was briefly but vigorously vortexed. The samples were centrifuged at $2000 \mathrm{~g}$ for $10 \mathrm{~min}$ and the absorbance of the supernatant was determined with a DU 640 spectrophotometer $(<1.8-\mathrm{nm}$ slit width; Beckmann Instruments, Inc., Fullerton, CA, USA) using a 200- $\mu$ l quartz cuvette (Sigma). The absorption spectrum peaks for the white, yellow, red, and blue microspheres were obtained at $370,448,530$, and $672 \mathrm{~nm}$, respectively. The absorbances were corrected for overlapping using a matrix inversion technique (Dye-Trak ${ }^{\circledR}$ matrix macro-inversion for Excel; Triton Technology, San Diego, CA, USA). The minimum acceptable absorbance was 0.010 absorbance units (AU).

For each infusion, the tissue flow rates were calculated according to (4):

$\mathrm{Qt}=\mathrm{At}(\mathrm{Qb} \div \mathrm{Ab})$

where Qt and Qb represent flow in the sample tissue and in the reference blood, respectively; At and Ab represent the peak absorbance (AU) of the tissue sample and of the reference blood, respectively. The $\mathrm{Qb}$, in $\mathrm{ml} /$ min, was calculated by:

$\mathrm{Qb}=\frac{\text { Reference blood sample weight } \div 1.05 \mathrm{~g} / \mathrm{ml}}{\text { Reference blood sample volume } \div 0.5 \mathrm{ml} / \mathrm{min}}$

where $1.05 \mathrm{~g} / \mathrm{ml}$ is the specific gravity of blood and $0.5 \mathrm{ml} / \mathrm{min}$ the withdrawal rate.

Blood flow rates were divided by tissue 
Figure 1. Spectrum of four different colors of the dye solution obtained from microspheres in heart, lungs, right and left kidneys, soleus and gastrocnemius muscles from one rat. Arrows indicate the wavelength of the absorbance of white $(370 \mathrm{~nm})$, yellow $(448 \mathrm{~nm})$, red $(530 \mathrm{~nm})$, and blue $(672 \mathrm{~nm})$ colors for the calculation of tissue blood flow. weights to yield $\mathrm{ml} \mathrm{min}^{-1} \mathrm{~g}^{-1}$. CO was calculated by the following formula (11):

Total number of injected microspheres $\mathrm{x}$ reference rate $(0.5 \mathrm{ml} / \mathrm{min})$

Number of microspheres in the reference blood sample

Data are reported as mean \pm SEM, and the paired $t$-test and repeated measure ANOVA were used to compare data, followed by the Tukey test. A P value of 0.05 was considered to be statistically significant.

Figure 1 shows the spectra of the four different colors of microspheres (red, white, yellow, and blue, injected at rest, post-aAVP, post-losartan, and post-hexamethonium, respectively) presented in the dye solution obtained from individual samples of heart, right
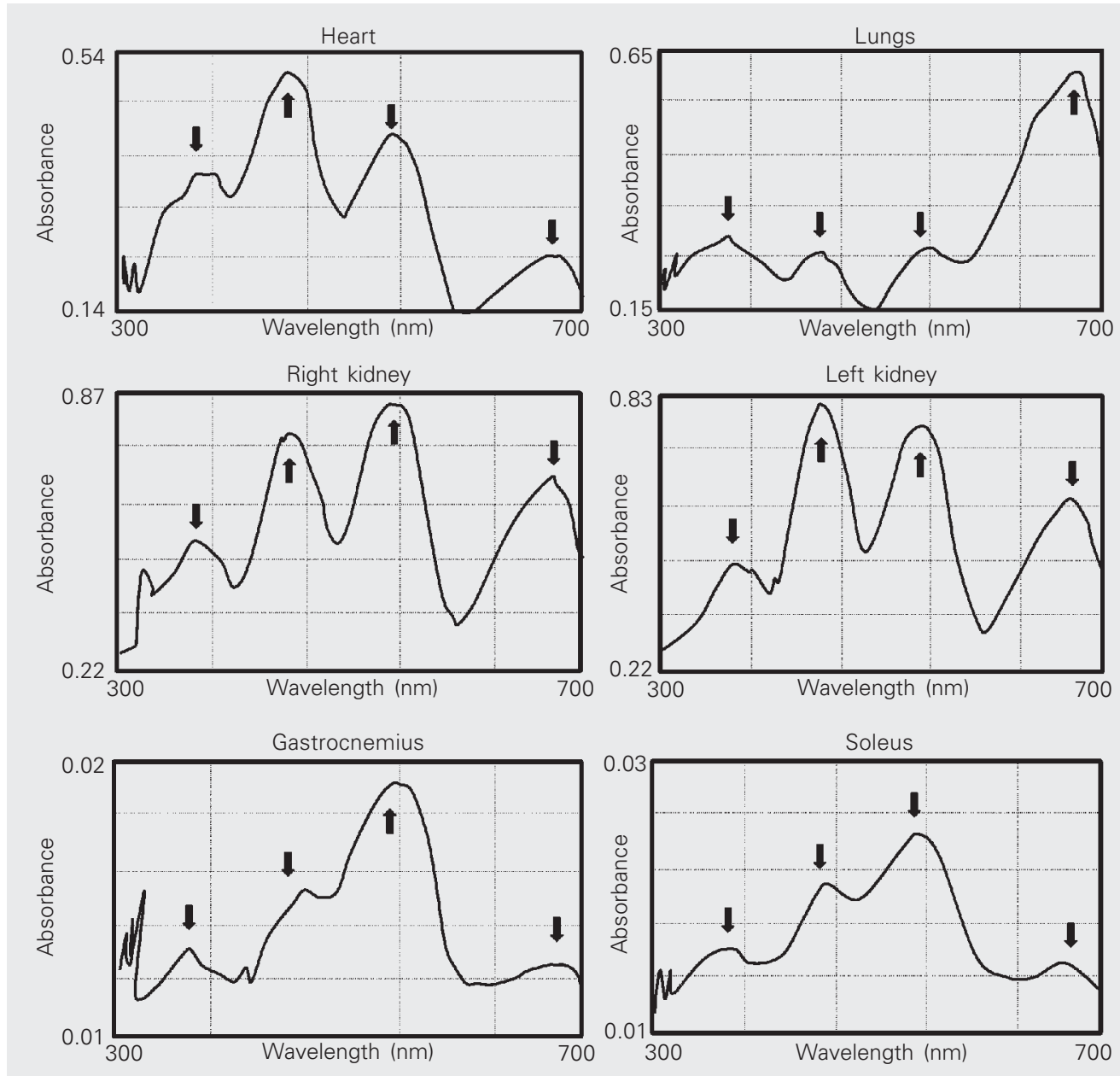

and left kidneys and soleus muscle. These results showed individual absorption spectrum peaks with no interference for the white $(370 \mathrm{~nm})$, yellow $(448 \mathrm{~nm})$, red $(530 \mathrm{~nm})$, and blue $(672 \mathrm{~nm})$ components of the microspheres in the heart, lungs, kidneys, and the gastrocnemius and soleus muscle tissues, demonstrating the ease of accurately measuring each color used. Similar spectrum peaks were obtained for these tissues and for the liver and triceps brachial muscle in all rats studied.

After blockade of each vasopressor system, AP was recorded $(10 \mathrm{~min})$ and the microspheres were infused. Before starting each protocol step, a 25-min interval was allowed to elapse between the use of the different blockers for blood pressure to re- 
turn to basal values. Blockade of the vasopressin $(108 \pm 2$ to $102 \pm 2 \mathrm{mmHg})$ and renin-angiotensin $(110 \pm 2$ to $103 \pm 3 \mathrm{mmHg})$ systems induced slight reductions in MAP $(-5.7 \pm 0.5$ and $-7.8 \pm 1.2 \mathrm{mmHg}$, respectively). However, hexamethonium administration induced a significant $52 \pm 3 \mathrm{mmHg}$ fall in MAP (106 \pm 3 to $53 \pm 3 \mathrm{mmHg})$. There were no significant changes in $\mathrm{HR}$ after aAVP ( $316 \pm 10$ to $320 \pm 10 \mathrm{bpm})$ or losartan (322 \pm 11 to $336 \pm 11 \mathrm{bpm}$ ), while sympathetic blockade induced a significant increase in HR ( $347 \pm 8$ to $371 \pm 6 \mathrm{bpm})$.

The CO and the regional blood flow measurements made during the neurohumoral study are presented in Table 1 . There were no significant changes in $\mathrm{CO}$ values after vasopressor system blockade. Vasopressin blockade increased blood flow in the lungs, liver and skeletal muscle. Losartan enhanced blood flow in the heart, lungs, kidneys, and skeletal muscle (gastrocnemius and soleus). Sympathetic inhibition by hexamethonium reduced kidney blood flow but left the other blood flows unchanged compared with basal values.

The aim of this study was to examine the efficacy of using nonradioactive $\mathrm{CM}$ for four repeated estimations of blood flow in the same rat. To reach this objective it was necessary to obtain accurate and reliable determinations of several color components in the same tissue. The combination of different numbers of four CM $(200,000$ white or blue or 150,000 red or yellow microspheres) using small amounts for colors that produce greatest absorption after dye extraction, associated with the use of reduced volume of DMF for dye extraction, permitted us to use four repeated estimates of $\mathrm{CO}$ and regional blood flow while evaluating different vasopressor systems in terms of blood pressure maintenance.

Indeed, $\mathrm{CM}$ as a tool to evaluate differences in blood flow is already validated $(4,6,8)$. The present experiments, using the rationale of previously tested changes in DMF volume for dye extraction $(4,8)$, as well as based on the minimum number of CM associated with its minimum absorbance (4), allow the determination of four different blood flow measurements in the same rat.

In fact, the half amount of DMF $(250 \mu \mathrm{l})$ used in the present study reduced the minimum number of spheres in the sample (97 red or yellow, 140 white or 245 blue microspheres) necessary to obtain a minimum absorbance of $0.01 \mathrm{AU}$. These changes in protocol allowed the use of exactly half the amount previously described in the literature (4). As a consequence, the minimum number of each color microsphere that would have to be systemically infused to measure flow in the different tissues was reduced, decreasing the risk to produce tissue embolism and hemodynamic perturbations (11). Moreover, Flaim et al. (12) have reported that infusion of up to 850,000 microspheres does not cause hemodynamic effects in rats. Although different investigators have used similar total amounts of microspheres to obtain acceptable absorbance in large tissues (heart, kidneys and lungs), only in our study did this procedure permit the analysis of changes in tissues with small weight (soleus muscle) or low blood flow (gastrocnemius muscle). This improvement overcomes in part the disadvantage of using this tech-

Table 1. Cardiac output and blood flow evaluations during vasopressor system blockade.

\begin{tabular}{|c|c|c|c|c|}
\hline Blood flow & Basal & aAVP & Losartan & Hexamethonium \\
\hline Cardiac output (ml/min) & $110 \pm 14$ & $142 \pm 22$ & $143 \pm 17$ & $86 \pm 12$ \\
\hline Heart $\left(\mathrm{ml} \mathrm{min} \mathrm{m}^{-1} \mathrm{~g}^{-1}\right)$ & $3.4 \pm 0.8$ & $4 \pm 1.1$ & $7.7 \pm 1.1^{*}$ & $3 \pm 0.6$ \\
\hline Lungs ( $\mathrm{ml} \mathrm{min-1} \mathrm{g}^{-1}$ ) & $1.8 \pm 0.2$ & $3.2 \pm 0.5^{*}$ & $3.6 \pm 0.7^{*}$ & $1.9 \pm 0.5$ \\
\hline Right kidney $\left(\mathrm{ml} \min ^{-1} \mathrm{~g}^{-1}\right)$ & $5.7 \pm 1$ & $5.2 \pm 1.3$ & $10.1 \pm 2^{*}$ & $2.9 \pm 0.7^{*}$ \\
\hline Left kidney ( $\mathrm{ml} \mathrm{min-1} \mathrm{g}^{-1}$ ) & $5.5 \pm 1$ & $5.9 \pm 1.1$ & $10 \pm 1.8^{*}$ & $2.7 \pm 0.7^{*}$ \\
\hline Liver $\left(m / \min ^{-1} \mathrm{~g}^{-1}\right)$ & $0.6 \pm 0.2$ & $1.3 \pm 0.2^{*}$ & $0.9 \pm 0.2$ & $0.5 \pm 0.1$ \\
\hline Brachial triceps $\left(\mathrm{ml} \min ^{-1} \mathrm{~g}^{-1}\right)$ & $0.8 \pm 0.2$ & $4.1 \pm 0.9^{*}$ & $2.1 \pm 0.8$ & $0.9 \pm 0.1$ \\
\hline Gastrocnemius ( $\mathrm{ml} \min ^{-1} \mathrm{~g}^{-1}$ ) & $0.4 \pm 0.08$ & $1 \pm 0.3^{*}$ & $0.7 \pm 0.1^{*}$ & $0.3 \pm 0.1$ \\
\hline Soleus ( $\mathrm{ml} \mathrm{min} \mathrm{m}^{-1} \mathrm{~g}^{-1}$ ) & $1.8 \pm 0.3$ & $6.4 \pm 1.5^{*}$ & $5.3 \pm 1.9 *$ & $1.6 \pm 0.3$ \\
\hline
\end{tabular}

Data are reported as means \pm SEM for 6 rats. aAVP $=$ vasopressin $V 1$ receptor antagonist.

${ }^{*} \mathrm{P}<0.05$ vs basal values (ANOVA). 
nique, which usually requires a larger number of CM than that required by the radioactive method to produce a minimum detectable signal.

In the present study, infusion and withdrawal rates were changed to allow measurements of a slight reduction in AP such as that observed with aAVP and/or losartan administration. The rates previously described by Hakkinen et al. (4) induced increased AP although it was established that to accurately evaluate small blood flow changes, microsphere infusion should not change hemodynamic parameters. In the present study, we used a spectrophotometer for colorimetric measurements instead of a microplate reader (4) or HPLC (8), which reduced the cost of the necessary equipment, but increased the overall time required for these measurements.

Heymann et al. (11) have provided a comprehensive review of the use and validation of the microsphere method. Recently, Prinzen and Bassingthwaighte (1) discussed the pros and cons of radioactive, colored and fluorescent microspheres. The CM technique allows the measurement of regional blood flow in a manner quite similar to that of radioactive microspheres $(4,7,8,13)$ while avoiding the disadvantage of radioisotope decay, limiting their lifetime, and the handling of radioactive materials. In a study that used fluorescent and CM simultaneously and compared them with radioactive microspheres, no differences were found in the accuracy of blood flow values obtained with the two types of non-radioactive spheres (7). In the present study, the basal $\mathrm{CO}$ and regional blood flow values were similar to values obtained by using radiolabeling and dye extraction methods in rats $(4,14)$. Moreover, the similar spectra and blood flows observed in the right and left kidneys during the four color evaluations provided evidence for good mixing and distribution of the microspheres in the circulation, since changes in kidney flow are used to analyze this qual- ity parameter (4).

The changes in MAP observed in the present experiment were similar to those obtained in a previous study $(10,15)$. The V1 blockade did not change basal AP values, probably because the increase in lung, liver and skeletal muscle blood flow was accompanied by a slight increase in CO. Similar findings were observed with losartan administration, with the increase in regional blood flow being associated with no changes in blood pressure. Indeed, Ullman et al. (16) have reported increased regional blood flow changes without concomitant effects on systemic hemodynamics.

The marked decrease in MAP observed after hexamethonium injection agreed with the data reported by Fink and Ploucha (17). However, these investigators reported that sympathetic blockade reduced blood pressure by vasodilation alone since no effect on $\mathrm{CO}$ was observed. The data reported in the present study did not show significant changes in regional blood flow after hexamethonium injection, except for the reduction in kidney blood flow. This reduction may be associated with decreased CO. However, the blood pressure decrease induced by sympathetic blockade produced only a nonsignificant reduction in CO (20\%), indicating that other changes in regions in which measurements were not performed should be considered.

Associated with this impaired reduction in blood pressure, hexamethonium administration induced an increase in HR, probably representing a baroreflex-mediated tachycardia induced by the marked decrease in AP. This HR increase is probably due to a vagal withdrawal, since sympathetic activity was blocked. Interestingly, the magnitude of the peak depressor response seems not to be associated with V1/AT1 receptor blockade since Santajuliana et al. (10) have previously demonstrated no changes in the depressor response with and without V1/AT1 blockade. 
The results of the present study demonstrate that four repeated blood flow measurements in the same rat with the use of four CM (white, yellow, red, and blue) can be performed accurately to detect small alterations in low blood flow tissues, suggesting that this technique is potentially useful for estimating blood flow in the same rats in at least four different situations. Using this approach, it was possible to describe changes in regional blood flow and $\mathrm{CO}$ induced by vasopressor system blockade in conscious rats.

\section{References}

1. Prinzen FW \& Bassingthwaighte JB (2000). Blood flow distribution by microsphere deposition methods. Cardiovascular Research, 45: 13-21.

2. Hale SL, Alker KJ \& Kloner RA (1988). Evaluations of nonradioactive, colored microspheres for measurements of regional myocardial blood flow in dogs. Circulation, 78: 428-434

3. Kowallik P, Schulz R, Guth BD, Schade A, Paffhausen W, Gross R \& Heusch G (1991). Measurement of regional myocardial blood flow with multiple colored micropheres. Circulation, 83: 974-982.

4. Hakkinen JP, Miller MW, Smith AH \& Knight DR (1995). Measurement of organ blood flow with coloured microspheres in the rat. Cardiovascular Research, 29: 74-79.

5. Baer RW, Payne BD, Verrier ED, Vlahakes GJ, Molodowicth PN, Uhlig PN \& Hoffman JI (1984). Increased number of myocardial blood flow measurements with radionuclide-labeled microspheres. American Journal of Physiology, 246 (Part 2): H418-H434.

6. Wieland W, Wouters PF, Van Aken H \& Flameng W (1993). Measurement of organ blood flow with coloured microspheres: a first time-saving improvement using automated spectrophotometry. In: Computers in Cardiology. IEEE Society Press, Los Alamitos, CA, USA, 691-694.

7. Hodeige D, de Pauw M, Eeghaute W, Weyne J \& Heyndrickx GR (1999). On the validity of blood flow measurement using colored microspheres. American Journal of Physiology, 276: H1150-H1158.

8. Mazoit JX, Le Guen R, Decaux A, Albaladejo P \& Samii K (1998). Application of HPLC to counting of colored microspheres in determination of regional blood flow. American Journal of Physiology, 274: $\mathrm{H} 1041-\mathrm{H} 1047$

9. De Angelis KLD, Gadonski G, Fang J, Dall'Ago P, Albuquerque VL, Peixoto LRA, Fernandes TG \& Irigoyen MC (1999). Exercise reverses peripheral insulin resistance in trained L-NAME-hyperten- sive rats. Hypertension, 34: 768-772.

10. Santajuliana D, Zukowska-Grojec Z \& Osborn JW (1997). Contribution of alpha- and beta-adrenoceptors and neuropeptide- $Y$ to autonomic dysreflexia. Clinical Autonomic Research, 5: 91-97.

11. Heymann MA, Payne BD, Hoffman JI \& Rudolph AM (1977). Blood flow measurements with radionuclide-labeled particles. Progress in Cardiovascular Diseases, 20: 55-79.

12. Flaim S, Nellis S, Toggart E, Drexler H, Kanda K \& Newman E (1984). Multiple simultaneous determination of hemodynamics and flow distribution in conscious rats. Journal of Pharmacological Methods, 11: 1-39.

13. Austin REJ, Hauck WM, Aldea GS, Blynn AE, Coggins DL \& Hoffman $\mathrm{JI}$ (1989). Quantitating error in blood flow measurements with radioactive microspheres. Journal of Applied Physiology, 257: H280H288.

14. Huang M, Manning Jr RD, LeBlanc MH \& Hester RL (1995). Overal hemodynamic studies after chronic inhibition of endothelial-derived nitric oxide in rats. American Journal of Hypertension, 8: 358-364.

15. Gravas H, Hatzinikolaou P, William GN, Margaret B \& Gravas (1981). Interaction of sympathetic nervous system with vasopressin and renin in the maintenance of blood pressure. Hypertension, 4: 400-405.

16. Ullman J, Eriksson S \& Rundgren M (2001). Effects of losartan, prazosin and vasopressin V1-receptor antagonist on renal and femoral blood flow in conscious sheep. Acta Physiologica Scandinavica, 17: 99-104

17. Fink GD \& Ploucha JM (1986). Contribution of the autonomic nervous system and vasopressin to elevated vascular resistance in the spontaneously hypertensive rats. Journal of Hypertension, 4 (Suppl 6): S594-S596. 